\title{
Bilateral Salpingo-0ophorectomy Compared to Gonadotropin-Releasing Hormone Agonists in Premenopausal Hormone Receptor-Positive Metastatic Breast Cancer Patients Treated with Aromatase Inhibitors
}

\section{Koung Jin Suh, MD ${ }^{1}$ \\ Se Hyun Kim, MD ${ }^{1}$ \\ Kyung-Hun Lee, MD2,3 \\ Tae-Yong Kim, MD2 \\ Yu Jung Kim, MD' \\ Sae-Won Han, MD2,3 \\ Eunyoung Kang, $\mathrm{MD}^{4}$ \\ Eun-Kyu Kim, MD ${ }^{4}$ \\ Kidong Kim, MD \\ Jae Hong No, MD \\ Wonshik Han, MD3,6 \\ Dong-Young Noh, MD,6 \\ Maria Lee, $\mathrm{MD}^{7}$ \\ Hee Seung Kim, $\mathrm{MD}^{6}$ \\ Seock-Ah Im, MD2,3 \\ Jee Hyun Kim, MD ${ }^{1}$}

*A list author's affiliations appears at the end of the paper.

\begin{abstract}
Purpose
Although combining aromatase inhibitors (Al) with gonadotropin-releasing hormone agonists $(\mathrm{GnRHa})$ is becoming more common, it is still not clear if $\mathrm{GnRHa}$ is as effective as bilateral salpingo-oophorectomy (BSO).
\end{abstract}

\section{Materials and Methods}

We retrospectively analyzed data of 66 premenopausal patients with hormone receptorpositive, human epidermal growth factor receptor 2-negative recurrent and metastatic breast cancer who had been treated with Als in combination with GnRHa or BSO between 2002 and 2015.

\section{Results}

The median patient age was 44 years. Overall, 24 (36\%) received BSO and 42 (64\%) received $\mathrm{GnRHa}$. The clinical benefit rate was higher in the BSO group than in the GnRHa group ( $88 \%$ vs. $69 \%, p=0.092$ ). Median progression-free survival (PFS) was longer in the BSO group, although statistical significance was not reached (17.2 months vs. 13.3 months, $p=0.245$ ). When propensity score matching was performed, the median PFS was 17.2 months for the BSO group and 8.2 months for the $\mathrm{GnRHa}$ group $(p=0.137)$. Multivariate analyses revealed that the luminal B subtype (hazard ratio, 1.67; 95\% confidence interval [Cl], 1.08 to 2.60; $p=0.022$ ) and later-line treatment ( $\geq$ third line vs. first line; hazard ratio, 3.24; $95 \% \mathrm{Cl}, 1.59$ to $6.59 ; p=0.001$ ) were independent predictive factors for a shorter PFS. Incomplete ovarian suppression was observed in a subset of GnRHa-treated patients whose disease showed progression, with E2 levels higher than $21 \mathrm{pg} / \mathrm{mL}$.

\section{Conclusion}

Both BSO and GnRHa were found to be effective in our Al-treated premenopausal metastatic breast cancer patient cohort. However, further studies in larger populations are needed to determine if $\mathrm{BSO}$ is superior to $\mathrm{GnRHa}$.

\footnotetext{
Correspondence: Jee Hyun Kim, MD, PhD Department of Internal Medicine, Seoul National University Bundang Hospital, Seoul National University College of Medicine, 82 Gumi-ro 173beon-gil, Bundang-gu, Seongnam 13620, Korea Tel: 82-31-787-7022 Fax: 82-31-787-4098

E-mail: jhkimmd@snu.ac.kr

Received September 24, 2016 Accepted February 1, 2017 Published Online February 27, 2017
}

\section{Key words}

Breast neoplasms, Premenopause, Aromatase inhibitors, Ovariectomy, Gonadotropin-releasing hormone 


\section{Introduction}

Although bilateral salpingo-oophorectomy (BSO) has been used as a therapeutic option in hormone receptor (HR)-positive premenopausal women, this method has largely been replaced with suppression of ovarian function via gonadotropin-releasing hormone agonist (GnRHa). Combination therapy using the aromatase inhibitors (AI) and GnRHa is an increasingly common strategy in current clinical practice [1]. However, only a few published studies have directly compared the effectiveness of primary BSO and GnRHa therapy or their efficacy when combined with AIs in premenopausal patients. Taylor et al. [2] reported that both event-free survival and overall survival (OS) were similar for goserelin and ovariectomy. However, their study was found to be underpowered due to poor accrual (a power of 0.22 to rule out a $50 \%$ improvement by one treatment). Additionally, no comparative studies of premenopausal patients have compared the efficacy of BSO treatment with that of GnRHa. However, several studies have evaluated the efficacy of $\mathrm{AI} /$ goserelin combination therapy in premenopausal metastatic breast cancer patients with clinical benefit rates (CBR) ranging from $65.9 \%$ to $75 \%$ and median progression-free survival (PFS) ranging from 7.3 to 13 months [3-8].

There are definite concerns associated with GnRHa and incomplete ovarian suppression. Furthermore, the addition of AIs may lead to an increase in estrogen synthesis and therefore have a negative impact on treatment outcomes. A study investigating the effects of the adjuvant leuprorelin in premenopausal patients revealed a hormonal escape rate of $7 \%$, with the cutoff estradiol (E2) value being $30 \mathrm{pg} / \mathrm{mL}$ [9]. Another recent analysis of patients who had received adjuvant triptorelin and exemestane/tamoxifen revealed that one-third of patients had an E2 level higher than $2.72 \mathrm{pg} / \mathrm{mL}$ [10]. This is a strict threshold which indicates that E2 levels in these patients were inconsistent with postmenopausal levels in AI-treated patients, suggesting that ovarian function recovery is still present in GnRHa-treated patients. Accordingly, ensuring complete ovarian suppression is a vital issue and increased efforts should be made to determine the most efficacious method of ovarian suppression. In this study, we aimed to assess the efficacy of AIs in premenopausal metastatic/recurrent breast cancer patients who were subjected to two different ovarian function suppression (OFS) modalities: GnRHa and BSO.

\section{Materials and Methods}

\section{Study population}

We retrospectively analyzed a database of consecutive recurrent/metastatic breast cancer patients who were treated with AIs and either GnRHa or BSO between January 2002 and December 2015 at Seoul National University Hospital (SNUH) and Seoul National University Bundang Hospital (SNUBH). The inclusion criteria was as follows: (1) pathologically confirmed breast cancer; (2) positive estrogen receptor (ER) and / or progesterone receptor (PR) expression; (3) premenopausal state; (4) initial stage IV or recurrence after curative surgery; (5) use of oral letrozole ( $2.5 \mathrm{mg} /$ day) or oral anastrozole ( $1 \mathrm{mg} /$ day) as palliative endocrine therapy; and (6) OFS with BSO or GnRHa injected subcutaneously every 4 weeks. Patients without evaluable disease were excluded. Premenopausal status was defined by regular menstruation periods or serum estradiol (E2) levels and/or follicle-stimulating hormone $(\mathrm{FSH})$ levels within the premenopausal range. E2 and FSH levels were determined via chemiluminescent microparticle immunoassay, which was conducted using the ARCHITECT i2000 Immunoassay Analyzer (Abbott Diagnostics, Abbott Park, IL). The measurement range was 10 to $5,000 \mathrm{pg} / \mathrm{mL}$ for E2 and 0 to $750 \mathrm{mIU} / \mathrm{mL}$ for FSH. Premenopausal levels were defined (based on the assay reference ranges) as an FSH value less than 26.72 $\mathrm{mIU} / \mathrm{mL}$ and an E2 value greater than $21 \mathrm{pg} / \mathrm{mL}$. For patients who underwent $\mathrm{BSO}$, premenopausal status before BSO was used as a surrogate.

Immunohistochemical staining for ER and PR expression was categorized as positive when $\geq 1 \%$ of the tumor cells were stained according to the 2010 American Society of Clinical Oncology (ASCO)/College of American Pathologists (CAP) guidelines [11]. Human epidermal growth factor receptor 2 (HER2) status was assessed by either immunohistochemical analysis or gene amplification levels, which were obtained using fluorescence in situ hybridization. Immunohistochemical expression of HER2 was assessed based on the 2013 ASCO/CAP guidelines [12]. We excluded patients with HER2-positive tumors. Intrinsic subtypes were adopted from the 2011 St. Gallen Consensus Panel [13], with luminal A subtype characterized by its hormone receptor-positive phenotype $(\mathrm{HR}[+])$, HER2(-) and low Ki-67 $(<14 \%)$ and luminal B subtype defined by $\mathrm{HR}(+)$, HER2(-), and a high Ki-67 $(\geq 14 \%)$.

Clinical data were retrieved from patient medical records. The Institutional Review Boards at SNUH and SNUBH approved this study (B-1603/338-108), and it was conducted in accordance with the Declaration of Helsinki. 
Table 1. Patient characteristics

\begin{tabular}{|c|c|c|c|c|}
\hline \multirow{2}{*}{ Variable } & \multirow{2}{*}{$\begin{array}{c}\text { No. } \\
(n=66)\end{array}$} & \multicolumn{2}{|c|}{ OFS modality } & \multirow{2}{*}{ p-value } \\
\hline & & BSO $(n=24)$ & GnRHa $(n=42)$ & \\
\hline Age at AI treatment (yr) & & $47(36-53)$ & $43(29-56)$ & 0.019 \\
\hline \multicolumn{5}{|l|}{ Pathology } \\
\hline IDC & 61 & $22(92)$ & $39(93)$ & 0.860 \\
\hline Others & 5 & $2(8)$ & $3(7)$ & \\
\hline \multicolumn{5}{|l|}{ Intrinsic subtype by IHC } \\
\hline Luminal A & 30 & $13(54)$ & $17(41)$ & 0.498 \\
\hline Luminal B, HER2 negative & 24 & $8(33)$ & $16(38)$ & \\
\hline Unknown & 12 & $3(13)$ & $9(21)$ & \\
\hline \multicolumn{5}{|l|}{ Disease status at AI treatment } \\
\hline MBC at primary diagnosis & 26 & $8(33)$ & $18(43)$ & 0.446 \\
\hline Recurred & 40 & $16(67)$ & $24(57)$ & \\
\hline Curative operation & 40 & $16(67)$ & $24(57)$ & 0.446 \\
\hline Neoadjuvant/Adjuvant chemotherapy & 38 & $14(58)$ & $24(57)$ & 0.400 \\
\hline Radiation therapy & 26 & $11(69)$ & $15(63)$ & 0.692 \\
\hline Adjuvant hormone therapy & 37 & $15(63)$ & $22(52)$ & 0.714 \\
\hline Tamoxifen & 28 & $12(50)$ & $16(38)$ & \\
\hline Tamoxifen+GnRHa & 8 & $3(13)$ & $5(12)$ & \\
\hline$A I^{a)}$ & 1 & 0 & $1(2)$ & \\
\hline RFI (mo) & & $42.5(16.8-102.0)$ & $45.0(10.5-110.7)$ & 0.986 \\
\hline \multicolumn{5}{|l|}{ Previous palliative chemotherapy } \\
\hline No & 35 & $16(67)$ & $19(45)$ & 0.093 \\
\hline Yes & 31 & $8(33)$ & $23(55)$ & \\
\hline \multicolumn{5}{|l|}{ Previous palliative endocrine therapy } \\
\hline No & 34 & $12(50)$ & $22(52)$ & 0.852 \\
\hline Yes & 32 & $12(50)$ & $20(48)$ & \\
\hline Tamoxifen & 12 & $3(13)$ & $9(21)$ & \\
\hline Tamoxifen+GnRHa & 15 & $5(21)$ & $10(24)$ & \\
\hline GnRHa & 3 & $3(13)$ & 0 & \\
\hline $\mathrm{AI}^{\mathrm{a})}$ & 2 & $1(4)$ & $1(2)$ & \\
\hline \multicolumn{5}{|l|}{ Aromatase inhibitor } \\
\hline Letrozole & 61 & $24(100)$ & $37(88)$ & 0.150 \\
\hline Anastrozole & 5 & 0 & $5(12)$ & \\
\hline \multicolumn{5}{|l|}{ Extent of disease at AI treatment } \\
\hline Bone and soft tissue only & 27 & $10(42)$ & $17(41)$ & 0.925 \\
\hline Visceral metastasis & 39 & $14(58)$ & $25(59)$ & \\
\hline \multicolumn{5}{|l|}{ Line of $\mathrm{AI}$ as palliative treatment } \\
\hline 1st line & 20 & $9(38)$ & $11(26)$ & 0.187 \\
\hline 2nd line & 26 & $11(46)$ & $15(36)$ & \\
\hline$\geq 3$ rd line & 20 & $4(17)$ & $16(38)$ & \\
\hline
\end{tabular}

Values are presented as median (range) or number (\%). OFS, ovarian function suppression; BSO, bilateral salpingoophorectomy; GnRHa, gonadotropin-releasing hormone agonist; AI, aromatase inhibitor; IDC, infiltrating ductal carcinoma; IHC, immunohistochemistry; HER2, human epidermal growth factor receptor 2; MBC, metastatic breast cancer; RFI, relapse-free interval. a) AI due to chemotherapy-induced amenorrhea. 


\section{Treatment and patient evaluation}

Chest computed tomography scans (with or without the abdominopelvis) were performed every 8 to 12 weeks as a routine clinical procedure, and additionally when needed, to confirm patient response and assess disease progression. All responses were defined according to the revised Response Evaluation Criteria in Solid Tumors ver. 1.1. PFS was defined as the interval from the first day of AI treatment to either the date of disease progression or death. The objective tumor response rate (ORR) was defined as the total proportion of patients who achieved complete response (CR) or partial response (PR). CBR was defined as the percentage of patients with CR, PR, or stable disease (SD) after 6 months.

\section{Statistical analysis}

The chi-square test was used to evaluate associations between clinicopathological attributes based on OFS modality. PFS was calculated using the Kaplan-Meier method and the values were compared using the log-rank test. Univariate Cox proportional hazard regression (PHR) analyses were performed to evaluate the predictive value of each variable, and those found to be significant upon univariable analysis were introduced into the multivariable Cox PHR model for disease-free survival (DFS). Because of the retrospective nature of this study, we conducted propensity score matching to evaluate the efficacy of BSO and GnRHa. The propensity for each patient to undergo BSO or receive GnRHa was scored using multivariable logistic regression based on three variables that affected the PFS. All tests were two-sided, and a p-value of less than 0.05 was used to indicate statistical significance. All analyses were performed using SPSS ver. 21 (IBM Corp., Armonk, NY) and GraphPad Prism 5 (GraphPad Software Inc., La Jolla, CA) based on data collected through July 2016.

\section{Results}

\section{Patient characteristics}

The database identified 66 premenopausal patients with HR-positive recurrent or metastatic breast cancer who had been treated by $\mathrm{AI}$ and GnRHa or BSO combination therapy. The clinical characteristics of the 66 patients are shown in Table 1. The median follow-up period was 23.2 months (range, 2.9 to 84.0 months) and the median patient age at the time of treatment was 44 years. A total of 24 patients $(36 \%)$ received $\mathrm{BSO}$, while $42(64 \%)$ received GnRHa. In BSO group, the median time between $\mathrm{BSO}$ and initiation of $\mathrm{AI}$ was 0.5 months (range, 0 to 48.9 months). Only four patients underwent BSO longer than 6 months before AI treatment $(7.5,12.5,29.8$, and 48.9 months, respectively). Most patients had invasive ductal carcinomas ( $\mathrm{n}=61$ ), although two patients had invasive lobular carcinomas and three had unclassified metastatic carcinomas. The intrinsic subtype, which was identified by immunohistochemistry (IHC), could be determined for 54 patients, with 30 $(45 \%)$ having luminal a subtype and 24 luminal B subtype $(36 \%)$. Twenty-eight patients received tamoxifen and eight patients received tamoxifen plus GnRHa as adjuvant endocrine treatment. The median relapse-free interval was similar in the BSO group and the GnRHa group (42.5 months in the BSO group vs. 45.0 months in the GnRHa group, $\mathrm{p}=0.986$ ). A total of 31 patients $(47 \%)$ received prior palliative chemotherapy, while $32(48 \%)$ received prior palliative endocrine therapy.

\section{Treatment outcomes and subgroup analyses}

In the BSO group, six patients achieved PR (25\%) and 16 reached SD $(67 \%)$, with $15(63 \%)$ having SD for a period longer than 6 months. In the GnRHa group, one patient achieved CR $(2 \%)$, six reached PR $(14 \%)$, and 29 patients experienced SD $(69 \%)$, with $22(50 \%)$ having SD for longer than 6 months. The ORR was similar between groups (25\% for BSO vs. $16 \%$ for $\mathrm{GnRHa}, \mathrm{p}=0.413$ ), while the CBR was higher in the BSO group than in the GnRHa group ( $88 \%$ vs. $69 \%$, p=0.092). Median PFS was shorter in the GnRHa group (median, 17.2 months vs. 13.3

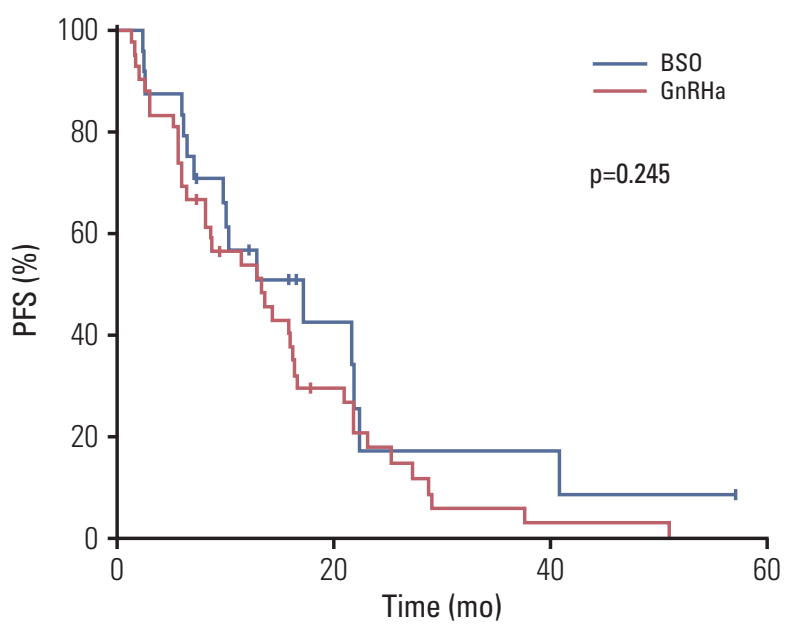

Fig. 1. Kaplan-Meier plots of progression-free survival (PFS) based on ovarian function suppression modality. $\mathrm{BSO}$, bilateral salpingo-oophorectomy; GnRHa, gonadotropin-releasing hormone agonists. 
Table 2. Univariate and multivariate analysis on PFS

\begin{tabular}{|c|c|c|c|c|}
\hline \multirow{2}{*}{ Variable } & \multicolumn{2}{|c|}{ Univariate } & \multicolumn{2}{|c|}{ Multivariate } \\
\hline & HR $(95 \%$ CI) & p-value & HR $(95 \%$ CI) & p-value \\
\hline \multicolumn{5}{|l|}{ Age at AI (yr) } \\
\hline $20-39$ & 1 & & - & \\
\hline $40-49$ & $0.95(0.50-1.77)$ & 0.859 & - & \\
\hline$\geq 50$ & $1.05(0.42-2.62)$ & 0.918 & - & - \\
\hline \multicolumn{5}{|l|}{ OFS modality } \\
\hline $\mathrm{BSO}$ & 1 & & - & \\
\hline GnRHa & $1.41(0.79-2.55)$ & 0.249 & - & - \\
\hline \multicolumn{5}{|l|}{ Pathology } \\
\hline IDC & 1 & & - & \\
\hline Others & $0.64(0.22-1.80)$ & 0.394 & - & - \\
\hline \multicolumn{5}{|l|}{ Intrinsic subtype } \\
\hline Luminal A & 1 & & 1 & \\
\hline Luminal B & $1.59(1.02-2.49)$ & 0.041 & $1.67(1.08-2.60)$ & 0.022 \\
\hline \multicolumn{5}{|l|}{ Disease status } \\
\hline MBC at primary Dx & 1 & & - & \\
\hline Recurred & $0.82(0.47-1.42)$ & 0.468 & - & - \\
\hline \multicolumn{5}{|l|}{ Extent of disease } \\
\hline Bone/Soft tissue & 1 & & - & \\
\hline Visceral & $1.48(0.85-2.58)$ & 0.168 & - & - \\
\hline \multicolumn{5}{|l|}{ AI } \\
\hline Letrozole & 1 & & - & \\
\hline Anastrozole & $2.39(0.83-6.88)$ & 0.107 & - & - \\
\hline \multicolumn{5}{|l|}{ Line of AI } \\
\hline 1st line & 1 & & 1 & \\
\hline 2nd line & $1.51(0.77-2.98)$ & 0.232 & $1.73(0.87-3.44)$ & 0.120 \\
\hline$\geq 3$ rd line & $3.01(1.48-6.13)$ & 0.002 & 3.24 (1.59-6.59) & 0.001 \\
\hline
\end{tabular}

PFS, progression-free survival; HR, hazard ratio; CI, confidence interval; AI, aromatase inhibitor; OFS, ovarian function suppression; BSO, bilateral salpingoophorectomy; GnRHa, gonadotropin-releasing hormone agonist; IDC, infiltrating ductal carcinoma; MBC, metastatic breast cancer; Dx, diagnosis.

months), although this difference was not statistically significant $(\mathrm{p}=0.245)$.

At the time of analysis, 39 patients in the GnRHa group experienced disease progression. Sixteen of these patients received cytotoxic chemotherapy, two were enrolled in clinical trials, and six had either died or were loss to follow-up. The remaining 16 patients underwent hormone therapy. Among them, eight patients (50\%) underwent BSO and received letrozole ( $n=2$; one patient experienced disease progression after 3.5 months of treatment, the other is still on treatment for 3 months), fulvestrant ( $\mathrm{n}=2$; one patient experienced disease progression after 12 months of treatment, the other is still on treatment for 8 months), and exemestane plus everolimus $(n=4$; patients experienced disease progression at 1.5, 10, 13, and 13 months, respectively). Additionally, three patients received GnRHa alone or in combination with fulvestrant or tamoxifen; however, all patients experienced disease progression within 2 months of treatment. Five patients became postmenopausal after receiving cytotoxic chemotherapy and then received endocrine treatment again (anastrozole [ $n=1]$, disease progressed after 4 months of treatment; exemestane [n=3], disease progressed after 2, 4, and 5 months, respectively; fulvestrant $[n=1]$, disease progressed after 1.5 months of treatment).

\section{Subgroup analysis based on extent of disease and line of treatment}

In the 39 patients who were diagnosed with visceral metastasis, the median PFS was longer in the BSO group than in the GnRHa group, although this was not statistically significant (17.2 months vs. 12.9 months, $p=0.261$ ) (Fig. 1). Additionally, CBR was higher in the BSO group than in the GnRHa group 


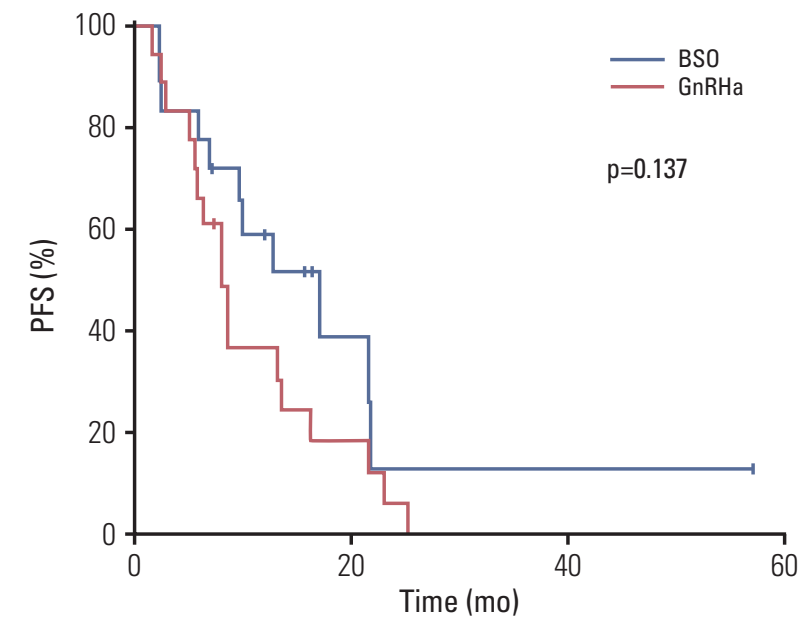

Fig. 2. Kaplan-Meier plots of progression-free survival (PFS) with propensity score matching according to ovarian function suppression modality. BSO, bilateral salpingooophorectomy; GnRHa, gonadotropin-releasing hormone agonists.

(85.7\% vs. $64.0 \%, \mathrm{p}=0.148)$. In patients with only bone and soft tissue diseases $(\mathrm{n}=27)$, the median PFS was similar between groups (12.9 months for BSO group vs. 14.3 months for GnRHa group, $\mathrm{p}=0.919$ ), while the CBR was $90 \%$ in the BSO group and $77 \%$ in the GnRHa group ( $\mathrm{p}=0.382$ ).

In the 20 patients who received AI plus OFS as the first-line treatment, the median PFS was 22.4 months in the BSO group $(\mathrm{n}=9)$ and 14.3 months in the GnRHa group $(\mathrm{p}=0.741)$. In patients who received second-line AI treatment $(n=26)$ the median PFS was 12.9 months in the BSO group $(\mathrm{n}=11)$ and 8.6 months in the GnRHa group $(n=15)(p=0.288)$. For patients who received AIs as a third-line, or above, treatment (BSO, $\mathrm{n}=4$; GnRHa, $\mathrm{n}=16$ ), the median PFS was shorter in the BSO group than the GnRHa group (2.5 months vs. 8.2 months, $\mathrm{p}=0.047)$.

\section{Univariate and multivariate analyses}

We conducted univariate and multivariate analyses to identify the predictive and prognostic importance of clinical characteristics. We also used these analyses to identify the OFS modality in premenopausal AI-treated breast cancer patients. Univariate Cox PHR analyses for PFS revealed no significant differences with respect to age, type of AI, pathological subtype, disease status, or the extent of disease at the time of AI treatment. However, PFS was shorter in luminal B subtype tumors $(\mathrm{p}=0.041)$, and in patients who received later-line AI treatments $(\mathrm{p}=0.002)$ (Table 2$)$.

Variables that were found to be significantly predictive in the univariate analyses were introduced into a multivariate Cox PHR model. Multivariate analyses revealed that luminal B subtype (hazard ratio [HR], 1.67; 95\% confidence interval

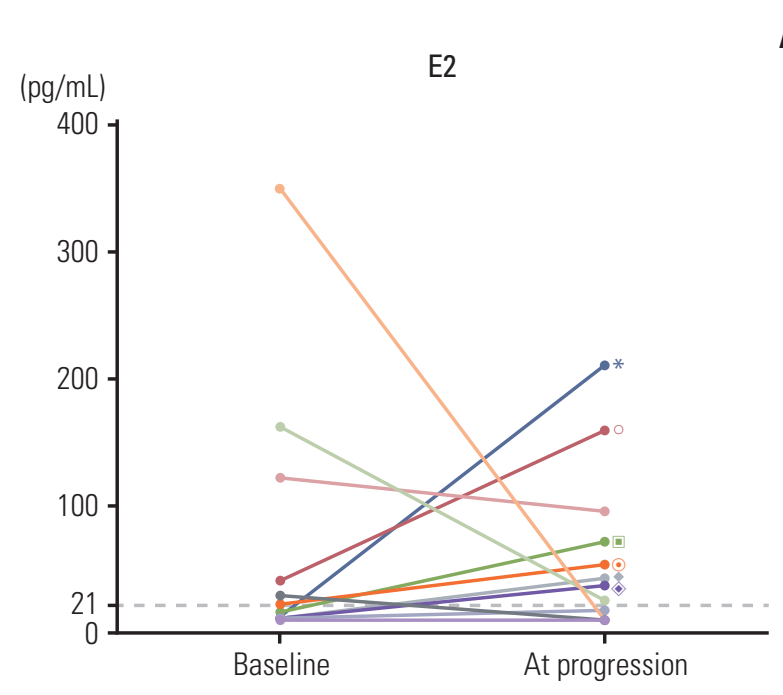

A

FSH

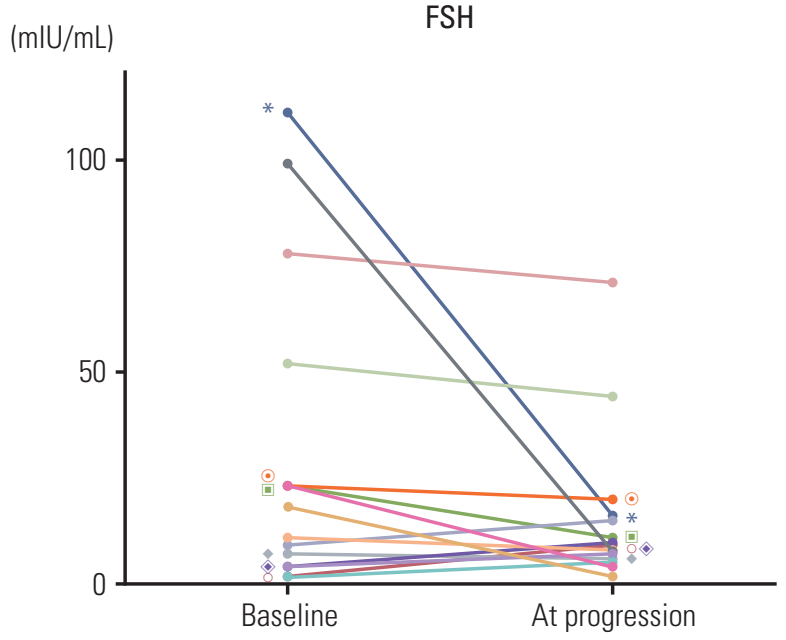

Fig. 3. The line charts show the individual changes in estradiol (E2) (A) and follicle-stimulating hormone (FSH) (B) between baseline and at progression in patients treated with aromatase inhibitors and gonadotropin-releasing hormone agonist. The value of same individuals on each graphs are marked with same symbols. Only patients with increased E2 levels compared to baseline are marked. 
[CI], 1.08 to 2.60; $\mathrm{p}=0.022$ ) and later-line AI treatment $(\geq$ third line vs. first line; HR, 3.24; 95\% CI, 1.59 to 6.59; $\mathrm{p}=0.001$ ) were significant independent predictive factors for shorter PFS (Table 2).

\section{Propensity score matching}

We used propensity score matching to compare the effects of BSO and GnRHa. To minimize the bias caused by nonrandom allocation of BSO and GnRHa, we developed a matching scheme that included variables shown to be associated with PFS in our analyses. We included the following domains: intrinsic subtype, the extent of disease at the time of AI treatment, and line of treatment. We then used propensity score matching to match the 18 patients (75\% of the relevant group) who received BSO to the 18 patients (43\% of the relevant group) who received GnRHa. With the exception of age, there were no significant differences in clinicopathological variables between groups (S1 Table). The median PFS was 17.2 months for the BSO group and 8.2 months for the GnRHa group $(\mathrm{p}=0.137)$ (Fig. 2). PFS did not differ significantly due to age (median PFS, 8.7 months for patients in their 20s and 30s vs. 12.9 months for patients in their 40s vs. 9.8 months for those in their 50s; $\mathrm{p}=0.840$ ). The ORR was $22.2 \%$ for the BSO group and $27.8 \%$ for the GnRHa group ( $p=1.000)$, while the CBR was $83.3 \%$ for the BSO group and $66.7 \%$ for the GnRHa group $(\mathrm{p}=0.443)$.

\section{Hormone study}

Of the 42 patients who were treated with $\mathrm{AI}$ and GnRHa, 36 $(86 \%)$ had at least one sample drawn for FSH and E2 measurement. S2 Table shows the serial E2 and FSH levels for these patients.

At the time of analysis, 39 patients in the GnRHa group experienced disease progression. Among these, 19 patients $(49 \%)$ were assessed for FSH and E2 levels at the time of disease progression. The results revealed that 16 of these patients had baseline FSH and E2 levels, while 10 had postmenopausal E2 levels (range, $<10$ to $17 \mathrm{pg} / \mathrm{mL}$ ), and an increase in serum E2 levels (into the premenopausal range) was observed in the remaining nine patients (range, 25 to 209 pg/mL). Fig. 3 shows the changes in FSH and E2 levels between baseline and at disease progression in patients treated with GnRHa plus AI.

Three patients underwent combined AI/GnRHa treatment at the time of analysis. Of these patients, two showed postmenopausal E2 levels, and one patient, whose treatment lasted for 7.4 months, showed an elevated E2 level at 3 months (87 $\mathrm{pg} / \mathrm{mL}$ at baseline to $105 \mathrm{pg} / \mathrm{mL}$ at 3 months).

\section{Discussion}

We found that both BSO and GnRHa function as active treatments in AI-treated $\mathrm{HR}(+)$ premenopausal advanced breast cancer patients. Both also had a statistically similar PFS in such patients. However, BSO had a numerically higher CBR (88\% vs. 69\%, p=0.092) and longer PFS (17.2 months vs. 13.3 months, $\mathrm{p}=0.245$ ), even after propensity score matching (median PFS, 17.2 months vs. 8.2 months; $p=0.137$ ). Assay of 19 of the 39 patients who showed disease progression in the GnRHa group for FSH and E2 expression at the time of disease progression revealed that approximately half of the tested GnRHa-treated patients $(n=9)$ showed evidence of incomplete ovarian suppression, with E2 expression similar to premenopausal levels.

Since the 1990s, BSO has been largely replaced by GnRHa in premenopausal $\mathrm{HR}(+)$ breast cancer patients. Early studies indicated that medical castration with GnRHa was well-tolerated, and that the ORR for such a treatment ranged between $36.4 \%$ and $45.9 \%$ in premenopausal and perimenopausal metastatic breast cancer patients $[14,15]$. This was followed by studies that directly compared oophorectomy to GnRHa. A randomized clinical trial using a $2 \times 2$ design compared surgical or radiation ovarian ablation and goserelin in one dimension and the usage of tamoxifen in the other. The results of this study revealed no significant differences in response rates after the $2 \times 2$ grouping. However, patients who were treated solely with goserelin $(n=22)$ had an ORR of $27.2 \%$ (standard error [SE], $18.6 \%$ ), while those who received oophorectomies (or ovarian ablation) $(n=15)$ had an ORR of $46.6 \%$ (SE, 25.3\%) [16]. Taylor et al. [2] reported that both event-free survival and OS were similar for goserelin treatment $(n=69)$ and oophorectomy $(n=67)$. However, their study was underpowered due to poor accrual (a power of 0.22 to rule out a $50 \%$ improvement by one treatment) [2].

Because of its comparable efficacy, lack of operative morbidity, and avoidance of psychological trauma, GnRHa has mostly replaced BSO, and has even been adopted as the standard method of OFS in AI or fulvestrant-treated premenopausal patients. Despite this, no studies conducted to date have directly compared the efficacy of BSO and GnRHa when used in combination with AIs. Nevertheless, studies have gauged the effectiveness of $\mathrm{AI}$ and GnRHa combination treatment. For example, Carlson et al. [4] conducted a phase 2 trial of anastrozole and goserelin in 35 premenopausal women, with a CBR of $71.9 \%$ and a median time to progression of 8.3 months (range, 2.1 to 63 months). The JMTO BC0801 study, which was a phase 2 trial that evaluated goserelin and anastrozole in 37 premenopausal women, found similar results, with a CBR of $62.2 \%$ and a median PFS of 7.3 months [7]. A parallel group study showed similar clinical outcomes 
between premenopausal patients who recei-ved letrozole plus goserelin and postmenopausal patients who received letrozole [5]. The efficacy of exemestane and GnRHa combination therapy has also been assessed $(n=44)$ and found to have an ORR of $38.6 \%$, a CBR of $65.9 \%$, and a median PFS of 13 months [8]. All of these studies concluded that AIs, when combined with GnRHa, are highly active and viable for inclusion in the standard sequence of endocrine therapies for premenopausal $\mathrm{HR}(+)$ metastatic breast cancer patients.

Since AIs are contraindicated in premenopausal patients because of the possible reactivation of ovarian functions, the capacity of GnRHa to induce OFS has become a far more important issue. Recent trials combining adjuvant AIs with GnRHa in premenopausal patients have attempted to look into this matter. Both the Suppression of Ovarian Function Trial (SOFT) and the combined analysis of the SOFT and the Tamoxifen and Exemestane Trial (TEXT) reported that, when compared to a combination of tamoxifen and OFS, combined exemestane plus OFS therapy showed higher 5-year DFS rates. However, this did not translate into an increase in OS in the exemestane/OFS group $[17,18]$. The Austrian Breast and Colorectal Cancer Study Group 12 (ABCSG-12) trial reported no significant differences in DFS rates between anastrozole/goserelin and tamoxifen/goserelin groups. Furthermore, a lower OS was observed in the anastrozole/goserelin group (HR, 1.63; 95\% CI, 1.05 to $1.45 ; \mathrm{p}=0.03$ ) [19]. A possible explanation for this could be that $\mathrm{AI} / \mathrm{GnRHa}$ treatment results in incomplete suppression of ovarian function [20]. The SOFT-EST Substudy demonstrated that one-third of patients who received adjuvant triptorelin and exemestane or tamoxifen had E2 levels higher than $2.72 \mathrm{pg} / \mathrm{mL}$, which is a strict threshold that indicates E2 levels inconsistent with postmenopausal AI-treated patients [10]. Similarly, Schmid et al. [9] reported an approximate hormonal escape rate of $7 \%$ with a cutoff E2 value of $30 \mathrm{pg} / \mathrm{mL}$ in premenopausal women $(n=209)$ who received adjuvant leuprorelin. In our study, although less than half of GnRHa-treated patients had samples drawn for a hormone study, nine out of 19 tested patients with progressive disease had E2 levels higher than $21 \mathrm{pg} / \mathrm{mL}$ (our defined premenopausal range). Collectively, these data seem to suggest that it is possible that GnRHa treatment results in incomplete ovarian suppression, which may translate into poorer outcomes when used in AI-treated premenopausal women.

There are multiple endocrine treatment options for postmenopausal advanced breast cancer patients, such as fulvestrant [21], everolimus combined with exemestane [22], and palbociclib combined with letrozole/fulvestrant [23,24]. Ovarian suppression is mandatory for premenopausal patients to take advantage of these multiple treatment options. For recurrent/metastatic $\mathrm{HR}(+)$ breast cancer patients, it is likely that lifelong ovarian suppression is needed until natural menopause is reached. The potential reversibility of ovarian function is less likely to be important for these patients. Because of advances in laparoscopic surgery, BSO can be performed via a minimally invasive approach with a relatively low complication rate (0\%-6.1\%) $[25,26]$. Therefore, permanent ovarian suppression via BSO might be advisable for patients who wish to avoid monthly GnRHa injections. In a recent survey of premenopausal metastatic breast cancer patients who received pharmacological ovarian suppression, seven of 13 patients stated that they would have chosen oophorectomy had it been initially offered. Inconvenience due to monthly goserelin was the main factor driving BSO preference [27]. Keeping this in mind, patients and physicians should rationally discuss and choose between a permanent surgical procedure and a reversible drug treatment.

Information describing cost-effectiveness and qualityof-life (QoL) may allow physicians and patients to make more informed decisions regarding therapeutic approaches. However, there is limited data directly comparing the toxicity and QoL factors associated with surgical and medical ovarian suppression in premenopausal patients. Boccardo et al. [16] reported a higher rate of side effects in patients treated with goserelin than in those treated with oophorectomy. Park et al. [5] reported that premenopausal women treated with $\mathrm{AI} /$ goserelin combination therapy showed more adverse events than AI-treated postmenopausal patients; specifically, hot flashes $(92.9 \%$ vs. $52.6 \%, \mathrm{p}=0.01)$, vaginal spotting $(28.6 \%$ vs. $2.6 \%, \mathrm{p}=0.002)$, weight gain ( $17.1 \%$ vs. $2.6 \%$, $\mathrm{p}=0.05)$, headaches ( $45.7 \%$ vs. $23.7 \%$, $\mathrm{p}=0.05)$, anorexia (31.4\% vs. $13.2 \%, \mathrm{p}=0.09)$, and vaginal dryness ( $34.3 \%$ vs. $13.2 \%, \mathrm{p}=0.05)$ were all more frequently observed in premenopausal patients than in postmenopausal patients. Moreover, one study demonstrated that oophorectomy was a cost effective method of ovarian suppression, with an incremental cost-effectiveness ratio of $\$ 7,849$. After 2 years of goserelin use, one-time laparoscopic surgery becomes a more cost-effective method of treatment, with the mean cost of annual goserelin therapy (in US dollars) being $\$ 2,536$ versus $\$ 3,966$ for a one-time laparoscopic ovarian ablation [28]. Further studies comparing the efficacy and safety of BSO and GnRHa in larger populations, along with QoL assessments and cost analyses, will help improve overall tumor management in premenopausal breast cancer patients.

Another important finding of our study is that later-line AI treatment and luminal B subtype were independent predictive factors for short PFS in premenopausal patients treated with AI. Since there are biological differences between the luminal A and B subtypes, a possibility of differences in their response to AI has been suggested. However, there is little data available regarding the relevance of biomarkers or breast cancer subtype to response to AIs. In a 
neoadjuvant study of AI in postmenopausal patients, there were no significant differences in AI response rates between luminal A and B subtypes [29]. In contrast, another study of ER-positive advanced breast cancer patients reported that higher Ki-67 levels were significantly associated with decreased time to AI treatment failure [30]. Our study is in line with the latter one in that luminal B subtype, which is distinguished from luminal A by having high Ki-67 index $(\geq 14)$, was found to be a negative predictive marker of response to AI treatment. Later-line treatment and a high Ki-67 index and/or luminal B subtype assessed by IHC might be useful in predicting AI response.

It should be noted that our study had several limitations. First, our study only involved a small number of patients from two Korean institutions. Therefore, it does not provide a global, accurate, or complete perspective of the treatment outcomes of AI-treated premenopausal patients. Second, our data is limited due to the heterogeneous time points used to determine patient hormone levels. Third, patients were treated with AIs at heterogeneous time points, which resulted in a higher proportion of patients in the GnRHa group having later-line treatments ( $\geq 3$ rd line). However, this heterogeneity was adjusted for the propensity score matched cohort, and the difference in median PFS was even more accentuated, favoring BSO over GnRHa. Finally, toxicity and QoL assessment could not be conducted owing to the retrospective design of this study.

In conclusion, we demonstrated that both $\mathrm{BSO}$ and $\mathrm{GnRHa}$ were found to be effective in premenopausal $\mathrm{HR}(+)$ AI-treated advanced breast cancer patients. However, BSO patients had a higher CBR and a longer PFS. Evidence of incomplete ovarian suppression was also observed in a subset of patients treated with GnRHa. These results may be useful when selecting the OFS modality for premenopausal advanced breast cancer patients. However, further studies in a larger population are needed to determine if BSO is truly superior to GnRHa.

\section{Electronic Supplementary Material}

Supplementary materials are available at Cancer Research and Treatment website (http:// www.e-crt.org).

\section{Conflicts of Interest}

T.Y. Kim has received a speaker honorarium from Roche. S.A. Im received a research fund from AstraZeneca, and has uncompensated advisory roles for Novartis, Spectrum and Hanmi. None of the other authors have any potential conflicts of interest.

\section{Acknowledgments}

This study was supported by the Basic Science Research Program through the National Research Foundation of Korea (NRF) funded by the Ministry of Science, ICT and future Planning (2015R1A2A2A01004655).

\section{Author Details}

${ }^{1}$ Department of Internal Medicine, Seoul National University Bundang Hospital, Seoul National University College of Medicine, Seongnam, ${ }^{2}$ Department of Internal Medicine, Seoul National University Hospital, Seoul National University College of Medicine, Seoul, ${ }^{3}$ Cancer Research Institute, Seoul National University College of Medicine, Seoul, Departments of ${ }^{4}$ Surgery and ${ }^{5}$ Obstetrics and Gynecology, Seoul National University Bundang Hospital, Seoul National University College of Medicine, Seongnam, Departments of ${ }^{6}$ Surgery and ${ }^{7}$ Obstetrics and Gynecology, Seoul National University Hospital, Seoul National University College of Medicine, Seoul, Korea

\section{References}

1. Rugo HS, Rumble RB, Macrae E, Barton DL, Connolly HK, Dickler MN, et al. Endocrine therapy for hormone receptorpositive metastatic breast cancer: American Society of Clinical Oncology Guideline. J Clin Oncol. 2016;34:3069-103.

2. Taylor CW, Green S, Dalton WS, Martino S, Rector D, Ingle JN, et al. Multicenter randomized clinical trial of goserelin versus surgical ovariectomy in premenopausal patients with receptor-positive metastatic breast cancer: an intergroup study. J Clin Oncol. 1998;16:994-9.

3. Forward DP, Cheung KL, Jackson L, Robertson JF. Clinical and endocrine data for goserelin plus anastrozole as second-line endocrine therapy for premenopausal advanced breast cancer.
Br J Cancer. 2004;90:590-4.

4. Carlson RW, Theriault R, Schurman CM, Rivera E, Chung CT, Phan SC, et al. Phase II trial of anastrozole plus goserelin in the treatment of hormone receptor-positive, metastatic carcinoma of the breast in premenopausal women. J Clin Oncol. 2010;28:3917-21.

5. Park IH, Ro J, Lee KS, Kim EA, Kwon Y, Nam BH, et al. Phase II parallel group study showing comparable efficacy between premenopausal metastatic breast cancer patients treated with letrozole plus goserelin and postmenopausal patients treated with letrozole alone as first-line hormone therapy. J Clin Oncol. 2010;28:2705-11. 
6. Yao S, Xu B, Li Q, Zhang P, Yuan P, Wang J, et al. Goserelin plus letrozole as first- or second-line hormonal treatment in premenopausal patients with advanced breast cancer. Endocr J. 2011;58:509-16.

7. Nishimura R, Anan K, Yamamoto Y, Higaki K, Tanaka M, Shibuta $\mathrm{K}$, et al. Efficacy of goserelin plus anastrozole in premenopausal women with advanced or recurrent breast cancer refractory to an LH-RH analogue with tamoxifen: results of the JMTO BC08-01 phase II trial. Oncol Rep. 2013;29:1707-13.

8. Wang J, Xu B, Yuan P, Ma F, Li Q, Zhang P, et al. Phase II trial of goserelin and exemestane combination therapy in premenopausal women with locally advanced or metastatic breast cancer. Medicine (Baltimore). 2015;94:e1006.

9. Schmid P, Untch M, Kosse V, Bondar G, Vassiljev L, Tarutinov $\mathrm{V}$, et al. Leuprorelin acetate every-3-months depot versus cyclophosphamide, methotrexate, and fluorouracil as adjuvant treatment in premenopausal patients with node-positive breast cancer: the TABLE study. J Clin Oncol. 2007;25:2509-15.

10. Bellet M, Gray KP, Francis PA, Lang I, Ciruelos E, Lluch A, et al. Twelve-month estrogen levels in premenopausal women with hormone receptor-positive breast cancer receiving adjuvant triptorelin plus exemestane or tamoxifen in the Suppression of Ovarian Function Trial (SOFT): The SOFT-EST Substudy. J Clin Oncol. 2016;34:1584-93.

11. Hammond ME, Hayes DF, Dowsett M, Allred DC, Hagerty KL, Badve S, et al. American Society of Clinical Oncology/College Of American Pathologists guideline recommendations for immunohistochemical testing of estrogen and progesterone receptors in breast cancer. J Clin Oncol. 2010;28:2784-95.

12. Wolff AC, Hammond ME, Hicks DG, Dowsett M, McShane $\mathrm{LM}$, Allison $\mathrm{KH}$, et al. Recommendations for human epidermal growth factor receptor 2 testing in breast cancer: American Society of Clinical Oncology/College of American Pathologists clinical practice guideline update. J Clin Oncol. 2013;31: 3997-4013.

13. Goldhirsch A, Wood WC, Coates AS, Gelber RD, Thurlimann B, Senn HJ, et al. Strategies for subtypes: dealing with the diversity of breast cancer: highlights of the St. Gallen International Expert Consensus on the Primary Therapy of Early Breast Cancer 2011. Ann Oncol. 2011;22:1736-47.

14. Kaufmann M, Jonat W, Kleeberg U, Eiermann W, Janicke F, Hilfrich J, et al. Goserelin, a depot gonadotrophin-releasing hormone agonist in the treatment of premenopausal patients with metastatic breast cancer. German Zoladex Trial Group. J Clin Oncol. 1989;7:1113-9.

15. Blamey RW, Jonat W, Kaufmann M, Bianco AR, Namer M. Goserelin depot in the treatment of premenopausal advanced breast cancer. Eur J Cancer. 1992;28A:810-4.

16. Boccardo F, Rubagotti A, Perrotta A, Amoroso D, Balestrero M, De Matteis A, et al. Ovarian ablation versus goserelin with or without tamoxifen in pre-perimenopausal patients with advanced breast cancer: results of a multicentric Italian study. Ann Oncol. 1994;5:337-42.

17. Pagani O, Regan MM, Walley BA, Fleming GF, Colleoni M, Lang I, et al. Adjuvant exemestane with ovarian suppression in premenopausal breast cancer. N Engl J Med. 2014;371:
107-18.

18. Francis PA, Regan MM, Fleming GF, Lang I, Ciruelos E, Bellet $\mathrm{M}$, et al. Adjuvant ovarian suppression in premenopausal breast cancer. N Engl J Med. 2015;372:436-46.

19. Gnant M, Mlineritsch B, Stoeger H, Luschin-Ebengreuth G, Knauer M, Moik M, et al. Zoledronic acid combined with adjuvant endocrine therapy of tamoxifen versus anastrozol plus ovarian function suppression in premenopausal early breast cancer: final analysis of the Austrian Breast and Colorectal Cancer Study Group Trial 12. Ann Oncol. 2015;26: 313-20.

20. Papakonstantinou A, Foukakis T, Rodriguez-Wallberg KA, Bergh J. Is estradiol monitoring necessary in women receiving ovarian suppression for breast cancer? J Clin Oncol. 2016;34: 1573-9.

21. Ellis MJ, Llombart-Cussac A, Feltl D, Dewar JA, Jasiowka M, Hewson $\mathrm{N}$, et al. Fulvestrant $500 \mathrm{mg}$ versus anastrozole $1 \mathrm{mg}$ for the first-line treatment of advanced breast cancer: overall survival analysis From the Phase II FIRST Study. J Clin Oncol. 2015;33:3781-7.

22. Baselga J, Campone M, Piccart M, Burris HA 3rd, Rugo HS, Sahmoud T, et al. Everolimus in postmenopausal hormonereceptor-positive advanced breast cancer. N Engl J Med. 2012;366:520-9.

23. Cristofanilli M, Turner NC, Bondarenko I, Ro J, Im SA, Masuda N, et al. Fulvestrant plus palbociclib versus fulvestrant plus placebo for treatment of hormone-receptor-positive, HER2-negative metastatic breast cancer that progressed on previous endocrine therapy (PALOMA-3): final analysis of the multicentre, double-blind, phase 3 randomised controlled trial. Lancet Oncol. 2016;17:425-39.

24. Finn RS, Martin M, Rugo HS, Jones SE, Im SA, Gelmon KA, et al. PALOMA-2: primary results from a phase III trial of palbociclib $(\mathrm{P})$ with letrozole $(\mathrm{L})$ compared with letrozole alone in postmenopausal women with ER+ / HER2-advanced breast cancer (ABC). J Clin Oncol. 2016;34(Suppl):Abstr 507.

25. Cho YJ, Kim ML, Lee SY, Lee HS, Kim JM, Joo KY. Laparoendoscopic single-site surgery (LESS) versus conventional laparoscopic surgery for adnexal preservation: a randomized controlled study. Int J Womens Health. 2012;4:85-91.

26. Angioni S, Pontis A, Sedda F, Zampetoglou T, Cela V, Mereu $\mathrm{L}$, et al. Single-port versus conventional multiport access prophylactic laparoscopic bilateral salpingo-oophorectomy in high-risk patients for ovarian cancer: a comparison of surgical outcomes. Onco Targets Ther. 2015;8:1575-80.

27. Hsieh AH, Kichenadasse G, Vatandoust S, Roy A, Sukumaran S, Karapetis CS, et al. Goserelin toxicities and preferences for ovarian suppression method in pre-menopausal women with breast cancer. Intern Med J. 2016;46:1153-9.

28. Hagemann AR, Zighelboim I, Odibo AO, Rader JS, Mutch DG, Powell MA. Cost-benefit of laparoscopic versus medical ovarian suppression in premenopausal breast cancer. Breast J. 2011;17:103-5.

29. Ellis MJ, Suman VJ, Hoog J, Lin L, Snider J, Prat A, et al. Randomized phase II neoadjuvant comparison between letrozole, anastrozole, and exemestane for postmenopausal women with 
estrogen receptor-rich stage 2 to 3 breast cancer: clinical and biomarker outcomes and predictive value of the baseline PAM50-based intrinsic subtype: ACOSOG Z1031. J Clin Oncol. 2011;29:2342-9.

30. Anderson H, Hills M, Zabaglo L, A'Hern R, Leary AF, Haynes
$\mathrm{BP}$, et al. Relationship between estrogen receptor, progesterone receptor, HER-2 and Ki67 expression and efficacy of aromatase inhibitors in advanced breast cancer. Ann Oncol. 2011;22:1770-6. 